\title{
On Some Further Generalizations of Strong Convergence in Probabilistic Metric Spaces Using Ideals
}

\author{
Pratulananda Das, ${ }^{1}$ Kaustubh Dutta, ${ }^{1}$ Vatan Karakaya, ${ }^{2}$ and Sanjoy Ghosal ${ }^{3}$ \\ ${ }^{1}$ Department of Mathematics, Jadavpur University, Kolkata 700032, India \\ ${ }^{2}$ Department of Mathematical Engineering, Yıldiz Technical University, Esenler, Istanbul 34210, Turkey \\ ${ }^{3}$ Department of Mathematics, Kalyani Government Engineering College, Kalyani, Nadia 741235, India
}

Correspondence should be addressed to Vatan Karakaya; vkkaya@yildiz.edu.tr

Received 21 June 2013; Accepted 5 September 2013

Academic Editor: Sung Guen Kim

Copyright (C) 2013 Pratulananda Das et al. This is an open access article distributed under the Creative Commons Attribution License, which permits unrestricted use, distribution, and reproduction in any medium, provided the original work is properly cited.

Following the line of (Das et al., 2011, Savas and Das, 2011), we make a new approach in this paper to extend the notion of strong convergence and more general strong statistical convergence (Şençimen and Pehlivan, 2008) using ideals and introduce the notion of strong $\mathscr{I}$ - and $\mathscr{I}^{*}$-statistical convergence and two related concepts, namely, strong $\mathscr{I}$-lacunary statistical convergence and strong $\mathscr{I}$ - $\lambda$-statistical convergence in a probabilistic metric space endowed with strong topology. We mainly investigate their interrelationship and study some of their important properties.

\section{Introduction and Background}

The usual idea of convergence is not enough to understand behaviours of those sequences which are not convergent. One of the approaches to include more sequences under purview is to consider those sequences which are convergent when restricted to some "big set of natural numbers." To accomplish this, the idea of convergence of real sequences was extended to statistical convergence by Fast [1], and it was further developed by several authors [2-4]. Recall that "asymptotic density" of a set $A \subseteq \mathbb{N}$ is defined as

$$
d(A)=\lim _{n \rightarrow \infty} \frac{1}{n}|\{k \leq n: k \in A\}|,
$$

provided that the limit exists, where $\mathbb{N}$ denotes the set of natural numbers and the vertical bar stands for cardinality of the enclosed set. The sequence $\left\{x_{n}\right\}_{n \in \mathbb{N}}$ of real is said to be statistically convergent to a real number $x$ if for each $\epsilon>0$,

$$
\lim _{n \rightarrow \infty} \frac{1}{n}\left|\left\{k \leq n:\left|x_{k}-x\right| \geq \epsilon\right\}\right|=0 .
$$

In another direction, a new type of convergence called lacunary statistical convergence was introduced and studied in [5]. More results related to this convergence can be found in [6]. The concept of $\lambda$-statistical convergence was introduced by Mursaleen in [7] as a further extension of statistical convergence. Afterward, in [8], Karakaya et al. defined statistical convergence of sequences of functions in intuitionistic fuzzy normed spaces and also used the concepts of lacunary and $\lambda$-statistical convergence for sequences of functions in these spaces in $[9,10]$. It must be mentioned in this context that some of the above mentioned convergence methods have applications in number theory, measure theory, fourier analysis, optimization, and many branches of mathematics.

The concepts of $\mathscr{I}$ and $\mathscr{I}^{*}$-convergence were introduced and investigated by Kostyrko et al. [11] as further generalizations of statistical convergence. They were much more general than other approaches as they were based on the very general notion of ideals of $\mathbb{N}$. In recent years, a lot of investigations have been done on ideal convergence and in some particular new approaches were made in $[12,13]$ to generalize the above mentioned convergences (for works on ideal convergence, see e.g., the papers [12-17] where many more references can be found).

On the other hand, the idea of probabilistic metric space was first introduced by Menger [18] in the name of "statistical 
metric space." In this theory, the concept of distance is probabilistic rather than deterministic. More precisely, the distance between two points $p, q$ is defined as a distribution function $F_{p q}$ instead of a nonnegative real number. For a positive number $t, F_{p q}(t)$ is interpreted as the probability that the distance between the points $p$ and $q$ is less than $t$. The theory of probabilistic metric spaces was brought to prominence by path breaking works of Schweizer et al. [19-22] and Tardiff [23] among others. Detailed theory of probabilistic metric space can be found in the famous book written by Schweizer and Sklar [24]. Several topologies can be defined on this space, but the topology that was found to be most useful is the "strong topology". Şençimen and Pehlivan [25] noted in their paper that as the strong topology is first countable and Hausdorff, it can be completely specified in terms of strong convergence of sequences. Since probabilistic metric spaces have many applications in applied mathematics, in order to provide a more general framework for applications, they have recently studied the statistical convergence and then strong ideal convergence in probabilistic metric spaces $[25,26]$ and also carried out further investigations in [27, 28].

In this paper, first of all, we introduce the notion of strong $\mathscr{I}$-statistical convergence in probabilistic metric spaces which happens to be more general than strong statistical convergence. We also introduce the concepts of strong $\mathscr{I}$ lacunary statistical convergence and strong $\mathscr{I}$ - $\lambda$-statistical convergence in probabilistic metric spaces and investigate some of their important properties, in particular their relations with strong $\mathscr{I}$-statistical convergence. As these concepts are more general than the concept of strong statistical convergence, we believe that they can extend the general framework introduced by [25] which in the future may enhance the applicability of strong convergence in probabilistic metric spaces.

\section{Preliminaries}

First, we recall some basic concepts related to the probabilistic metric spaces (in short PM spaces) (see [24]).

Definition 1. A nondecreasing function $F: \mathbb{R} \rightarrow[0,1]$ defined on $\mathbb{R}$ with $F(-\infty)=0$ and $F(\infty)=1$, where $\mathbb{R}=$ $[-\infty, \infty]$, is called a distribution function.

The set of all left continuous distribution functions over $(-\infty, \infty)$ is denoted by $\Delta$.

We consider the relation " $\leq$ " on $\Delta$ defined by $F \leq G$ if and only if $F(x) \leq G(x)$ for all $x \in \mathbb{R}$. It can be easily verified that the relation " $\leq$ " is a partial order on $\Delta$.

Definition 2. For any $a \in \mathbb{R}$, the unit step function at $a$ is denoted by $\varepsilon_{a}$ and is defined to be a function in $\Delta$ given by

$$
\varepsilon_{a}(x)= \begin{cases}0, & -\infty \leq x \leq a \\ 1, & a<x \leq \infty\end{cases}
$$

Definition 3. A sequence $\left\{F_{n}\right\}_{n \in \mathbb{N}}$ of distribution functions converges weakly to a distribution function $F$, and one writes $F_{n} \stackrel{w}{\rightarrow} F$ if and only if the sequence $\left\{F_{n}(x)\right\}_{n \in \mathbb{N}}$ converges to $F(x)$ at each continuity point $x$ of $F$.
Definition 4. The distance between $F$ and $G$ in $\Delta$ is denoted by $d_{L}(F, G)$ and is defined as the infimum of all numbers $h \epsilon$ $(0,1]$ such that the inequalities

$$
\begin{aligned}
& F(x-h)-h \leq G(x) \leq F(x+h)+h, \\
& G(x-h)-h \leq F(x) \leq G(x+h)+h
\end{aligned}
$$

hold for every $x \in(-1 / h, 1 / h)$.

Here, we are interested in the subset of $\Delta$ consisting of those elements $F$ that satisfy $F(0)=0$.

Definition 5. A distance distribution function is a nondecreasing function $F$ defined on $\mathbb{R}^{+}=[0, \infty]$ that satisfies $F(0)=0$ and $F(\infty)=1$ and is left continuous on $(0, \infty)$. $\Delta^{+}$.

The set of all distance distribution functions is denoted by

The function $d_{L}$ is clearly a metric on $\Delta^{+}$. The metric space $\left(\Delta^{+}, d_{L}\right)$ is compact and hence complete (see [29]).

Theorem 6 (see [24]). Let $F \in \Delta^{+}$be given. Then, for any $t>$ $0, F(t)>1-t$ if and only if $d_{L}\left(F, \varepsilon_{0}\right)<t$. >

Note 7. Geometrically, $d_{L}\left(F, \varepsilon_{0}\right)$ is the abscissa of the point of intersection of the line $y=1-x$ and the graph of $F$ (if necessary we add vertical line segment at the point of discontinuity).

Definition 8. A triangle function is a binary operation $\tau$ on $\Delta^{+}, \tau: \Delta^{+} \times \Delta^{+} \rightarrow \Delta^{+}$which is commutative, associative, and nondecreasing in each place and has $\varepsilon_{0}$ as identity.

Definition 9. A PM space is a triplet $(X, \mathfrak{F}, \tau)$ where $X$ is a nonempty set, $\mathfrak{F}$ is a function from $X \times X$ into $\Delta^{+}$, and $\tau$ is a triangle function. The following conditions for a PM space are satisfied for all $x, y, z \in X$ :

(1) $\mathfrak{F}(x, x)=\varepsilon_{0}$,

(2) $\mathfrak{F}(x, y) \neq \varepsilon_{0}$ if $x \neq y$,

(3) $\mathfrak{F}(x, y)=\mathfrak{F}(y, x)$,

(4) $\mathfrak{F}(x, z) \geq \tau(\mathfrak{F}(x, y), \mathfrak{F}(y, z))$.

In the sequel, we shall denote $\mathfrak{F}(x, y)$ by $F_{x y}$ and its value at $t$ by $F_{x y}(t)$. Throughout this paper $X$ shall represent the PM space $(X, \mathfrak{F}, \tau)$.

Definition 10. Let $X$ be a PM space. For $x \in X$ and $t>0$, the strong $t$-neighbourhood of $x$ is defined as the set

$$
\mathcal{N}_{x}(t)=\left\{y \in X: F_{x y}(t)>1-t\right\} .
$$

The collection $\mathfrak{N}_{x}=\left\{\mathcal{N}_{x}(t): t>0\right\}$ is called the strong neighbourhood system at $x$, and the union $\mathfrak{N}=\bigcup_{x \in X} \mathfrak{N}_{x}$ is called the strong neighbourhood system for $X$.

By Theorem 6, we can write

$$
\mathcal{N}_{x}(t)=\left\{y \in S: d_{L}\left(F_{x y}, \varepsilon_{0}\right)<t\right\} .
$$


If $\tau$ is continuous then the strong neighbourhood system $\mathfrak{N}$ determines a Hausdorff topology for $X$. This topology is called the strong topology for $X$.

Definition 11. Let $X$ be a PM space. Then, for any $t>0$ the subset $\mathfrak{U}(t)$ of $X \times X$ is given by $\mathfrak{U}(t)=\left\{(x, y): F_{x y}(t)>1-t\right\}$ and it is called the strong $t$-vicinity.

Theorem 12 (see [24]). Let $X$ be a PM space, and, $\tau$ be continuous. Then, for any $t>0$, there exists a $\eta>0$ such that $\mathfrak{U}(\eta)$ 。 $\mathfrak{U}(\eta) \subset \mathfrak{U}(t)$, where $\mathfrak{U}(\eta) \circ \mathfrak{U}(\eta)=\{(x, z)$ : for some $y,(x, y)$ and $(y, z) \in \mathfrak{U}(t)\}$.

Note 13. Under the hypothesis of Theorem 12, we can say that for any $t>0$ there is a $\eta>0$ such that $F_{x z}(t)>1-t$ whenever $F_{x y}(\eta)>1-\eta$ and $F_{y z}(\eta)>1-\eta$. Equivalently, it can be written as follows: for any $t>0$ there is an $\eta>0$ such that $d_{L}\left(F_{x z}, \varepsilon_{0}\right)<t$, whenever $d_{L}\left(F_{x y}, \varepsilon_{0}\right)<\eta$ and $d_{L}\left(F_{y z}, \varepsilon_{0}\right)<\eta$.

If $\tau$ is continuous in a PM space $X$, then the strong neighbourhood system $\mathfrak{N}$ determines a Kuratowski closure operation. It is termed as the strong closure. For any subset $A$ of $X$, the strong closure of $A$ is denoted by $\kappa(A)$ and is defined as

$$
\begin{array}{r}
\kappa(A)=\{x \in X: \text { for any } t>0, \text { there is a } \\
\left.y \in A \text { such that } F_{x y}(t)>1-t\right\} .
\end{array}
$$

Remark 14. Throughout the rest of the paper, we always assume that in a PM space $X$, the triangle function $\tau$ is continuous and $X$ is endowed with strong topology.

Definition 15. Let $X$ be a PM space. A sequence $\left\{x_{n}\right\}_{n \in \mathbb{N}}$ in $X$ is said to be strongly convergent to a point $\xi \in X$ if for any $t>0$ there exists a natural number $N$ such that $x_{n} \in \mathfrak{N}_{\xi}(t)$ whenever $n \geq N$. One writes $x_{n} \rightarrow \xi$ or $\lim _{n \rightarrow \infty} x_{n}=\xi$.

Similarly, a sequence $\left\{x_{n}\right\}_{n \in \mathbb{N}}$ in $X$ is called a strong Cauchy sequence if for any $t>0$ there exists a natural number $N$ such that $\left(x_{m}, x_{n}\right) \in \mathfrak{U}(t)$ whenever $m, n \geq N$.

Next, we recall some of the basic concepts related to the theory of $\mathscr{I}$-convergence, and we refer to [11] for more details.

Definition 16. Let $X$ be any nonempty set. Then, the family $\mathscr{I} \subseteq \mathscr{P}(X)$ is called an ideal in $X$ if

(1) $A, B \in \mathscr{I}$ imply $A \cup B \in \mathscr{I}$,

(2) if $A \in \mathscr{I}$ and $B \subseteq A$ then $B \in \mathscr{I}$.

Definition 17. Let $X$ be any nonempty set. The family $\mathscr{F} \subseteq$ $\mathscr{P}(X)$ is called a filter in $X$ if

(1) $\emptyset \notin \mathscr{F}$,

(2) $A, B \in \mathscr{F}$ imply $A \cap B \in \mathscr{F}$,

(3) if $A \in \mathscr{F}$ and $A \subseteq B$ then $B \in \mathscr{F}$.

If $\mathscr{I}$ is an ideal in $X$, then $\mathscr{F}(\mathscr{I})=\{X \backslash A: A \in \mathscr{I}\}$ is a filter in $X$, which is called the filter associated with the ideal $\mathscr{I}$. An ideal $\mathscr{I}$ in $X$ is called proper if and only if $X \notin \mathscr{I} . \mathscr{I}$ is called nontrivial if $\mathscr{I} \neq\{\emptyset\}$. An ideal is called an admissible ideal if it is proper and contains $\{x\}$ for all $x \in X$. In other words, it is called an admissible ideal if it is proper and contains all of its finite subsets.

Definition 18. An admissible ideal $\mathscr{I}$ is said to satisfy the condition (AP) if for every countable family of mutually disjoint sets $\left\{A_{1}, A_{2}, \ldots\right\}$ belonging to $\mathscr{I}$ there exists a countable family of sets $\left\{B_{1}, B_{2}, \ldots\right\}$ such that $A_{j} \Delta B_{j}$ is a finite set for every $j \in \mathbb{N}$ and $B=\bigcup_{j=1}^{\infty} B_{j} \in \mathscr{I}$.

Throughout the paper $\mathscr{I}$ stands for a nontrivial admissible ideal of $\mathbb{N}$, and $\mathscr{F}(\mathscr{I})$ is the filter associated with the ideal $\mathscr{I}$ of $\mathbb{N}$.

\section{Strong $\mathscr{I}$ - and $\mathscr{I}^{*}$-Statistical Convergence in PM Space}

In this section, we extend the concept of strong statistical convergence in PM spaces [25] via ideals and prove some associated results.

Definition 19. A sequence $\left\{x_{n}\right\}_{n \in \mathbb{N}}$ in a PM space $X$ is said to be strong statistically convergent to $x$ in $X$ if for $\epsilon>0$,

$$
\lim _{n \rightarrow \infty} \frac{1}{n}\left|\left\{k \leq n: x_{n} \notin \mathcal{N}_{x}(\epsilon)\right\}\right|=0 .
$$

Definition 20. A sequence $\left\{x_{n}\right\}_{n \in \mathbb{N}}$ of real numbers is said to be strong $\mathscr{I}$-statistically convergent to $x$ if for each $\epsilon>0$ and $\delta>0$,

$$
\left\{n \in \mathbb{N}: \frac{1}{n}\left|\left\{k \leq n:\left|x_{k}-x\right| \geq \epsilon\right\}\right| \geq \delta\right\} \in \mathscr{I} .
$$

In this case, we write $x_{n} \rightarrow x(S(\mathscr{I}))$.

We now introduce the definition of strong $\mathscr{I}$-statistical convergence in PM space. So, consider the following [12].

Definition 21. A sequence $\left\{x_{n}\right\}_{n \in \mathbb{N}}$ in a PM space $X$ is said to be strong $\mathscr{I}$-statistically convergent to $x$ if for each $\epsilon>0$ and $\delta>0$,

$$
\left\{n \in \mathbb{N}: \frac{1}{n}\left|\left\{k \leq n: x_{k} \notin \mathscr{N}_{x}(\epsilon)\right\}\right| \geq \delta\right\} \in \mathscr{I} .
$$

In this case, we write $x_{n} \rightarrow x\left(S_{\mathrm{PM}}(\mathscr{I})\right)$ and the class of all strong $\mathscr{I}$-statistically convergent sequences is simply denoted by $S_{\mathrm{PM}}(\mathscr{I})$.

Theorem 22. Let $X$ be a PM space, and, let $\tau$ be continuous. Then, the strong $\mathscr{I}$-statistical limit of a sequence in $X$ is unique.

Proof. If possible, let the sequence $\left\{x_{n}\right\}_{n \in \mathbb{N}}$ converge to two different limits $x_{1}$ and $x_{2}$ in $X$. Since $x_{1} \neq x_{2}$, we have $F_{x_{1} x_{2}} \neq \varepsilon_{0}$ and so there exists a positive real number $t$ such that $d_{L}\left(F_{x_{1} x_{2}}, \varepsilon_{0}\right)=t$. Choose $\eta>0$ such that $d_{L}\left(F_{x y}, \varepsilon_{0}\right)<\eta$ and $d_{L}\left(F_{y z}, \varepsilon_{0}\right)<\eta$ imply that $d_{L}\left(F_{x z}, \varepsilon_{0}\right)<t$. Since 
$x_{n} \rightarrow x_{1}\left(S_{\mathrm{PM}}(\mathscr{I})\right)$ and $x_{n} \rightarrow x_{2}\left(S_{\mathrm{PM}}(\mathscr{I})\right)$ for this $\eta>0$ and $0<\delta<1$,

$$
\begin{aligned}
& A=\left\{n \in \mathbb{N}: \frac{1}{n}\left|\left\{k \leq n: x_{k} \notin \mathcal{N}_{x_{1}}(\eta)\right\}\right|<\frac{\delta}{3}\right\} \in \mathscr{F}(\mathscr{I}), \\
& B=\left\{n \in \mathbb{N}: \frac{1}{n}\left|\left\{k \leq n: x_{k} \notin \mathcal{N}_{x_{2}}(\eta)\right\}\right|<\frac{\delta}{3}\right\} \in \mathscr{F}(\mathscr{I}) .
\end{aligned}
$$

Clearly, $A \cap B \neq \emptyset$. Let $m \in A \cap B$. Hence,

$$
\begin{aligned}
& \frac{1}{m}\left|\left\{k \leq m: x_{k} \notin \mathcal{N}_{x_{1}}(\eta)\right\}\right|<\frac{\delta}{3}, \\
& \frac{1}{m}\left|\left\{k \leq m: x_{k} \notin \mathcal{N}_{x_{2}}(\eta)\right\}\right|<\frac{\delta}{3},
\end{aligned}
$$

and consequently,

$$
\frac{1}{m} \mid\left\{k \leq m: x_{k} \notin \mathcal{N}_{x_{1}}(\eta) \text { or } x_{k} \notin \mathcal{N}_{x_{2}}(\eta)\right\} \mid<\delta<1 \text {. }
$$

Then, there exists some $k \leq m$ such that $x_{k} \in \mathcal{N}_{x_{1}}(\eta)$ and $x_{k} \in \mathcal{N}_{x_{2}}(\eta)$, that is, $d_{L}\left(F_{x_{k} x_{1}}, \varepsilon_{0}\right)<\eta$ and $d_{L}\left(F_{x_{k} x_{2}}, \varepsilon_{0}\right)<\eta$. Combining these two, we have $d_{L}\left(F_{x_{1} x_{2}}, \varepsilon_{0}\right)<t$, which is a contradiction. Hence, the limit is unique.

Theorem 23. Let $(X, \mathfrak{F}, \tau)$ be a PM space. If $\left\{x_{n}\right\}_{n \in \mathbb{N}}$ and $\left\{y_{n}\right\}_{n \in \mathbb{N}}$ are two sequences in $X$ such that $x_{n} \rightarrow x\left(S_{P M}(\mathscr{I})\right)$ and $y_{n} \rightarrow y\left(S_{P M}(\mathscr{I})\right)$, then one has $d_{L}\left(F_{x_{n} y_{n}}, F_{x y}\right) \rightarrow$ $0(S(\mathscr{I}))$.

Proof. For $t>0$ there exists a $\eta(t)>0$ such that $d_{L}\left(F_{x y}\right.$, $\left.F_{x^{\prime} y^{\prime}}\right)<t$ whenever $x^{\prime} \in \mathcal{N}_{x}(\eta)$ and $y^{\prime} \in \mathcal{N}_{y}(\eta)$. Now, assume that $x_{n} \rightarrow x\left(S_{\mathrm{PM}}(\mathscr{I})\right)$ and $y_{n} \rightarrow y\left(S_{\mathrm{PM}}(\mathscr{I})\right)$. Then, for any $t>0$, we have

$$
\begin{aligned}
\left\{n \in \mathbb{N}: d_{L}\left(F_{x_{n} y_{n}}, F_{x y}\right) \geq t\right\} \subseteq & \left\{n \in \mathbb{N}: x_{n} \notin \mathcal{N}_{x}(\eta)\right\} \\
& \cup\left\{n \in \mathbb{N}: y_{n} \notin \mathcal{N}_{y}(\eta)\right\} .
\end{aligned}
$$

Clearly,

$$
\begin{aligned}
\{n \in \mathbb{N}: & \left.\frac{1}{n}\left|\left\{k \leq n: d_{L}\left(F_{x_{k} y_{k}}, F_{x y}\right) \geq t\right\}\right| \geq \delta\right\} \\
\subseteq & \left\{n \in \mathbb{N}: \frac{1}{n}\left|\left\{k \leq n: d_{L}\left(F_{x_{k} x}, \varepsilon_{0}\right) \geq \eta\right\}\right| \geq \frac{\delta}{2}\right\} \\
\cup & \left\{n \in \mathbb{N}: \frac{1}{n}\left|\left\{k \leq n: d_{L}\left(F_{y_{k} y}, \varepsilon_{0}\right) \geq \eta\right\}\right| \geq \frac{\delta}{2}\right\} .
\end{aligned}
$$

Since $x_{n} \rightarrow x\left(S_{\mathrm{PM}}(\mathscr{I})\right)$ and $y_{n} \rightarrow y\left(S_{\mathrm{PM}}(\mathscr{I})\right)$, we have

$$
\begin{aligned}
& \left\{n \in \mathbb{N}: \frac{1}{n}\left|\left\{k \leq n: d_{L}\left(F_{x_{k} x}, \varepsilon_{0}\right) \geq \eta\right\}\right| \geq \frac{\delta}{2}\right\} \in \mathscr{I}, \\
& \left\{n \in \mathbb{N}: \frac{1}{n}\left|\left\{k \leq n: d_{L}\left(F_{y_{k} y}, \varepsilon_{0}\right) \geq \eta\right\}\right| \geq \frac{\delta}{2}\right\} \in \mathscr{I} .
\end{aligned}
$$

Therefore, union of these two sets also belongs to $\mathscr{I}$, and consequently $\left\{n \in \mathbb{N}:(1 / n)\left|\left\{k \leq n: d_{L}\left(F_{x_{k} y_{k}}, F_{x y}\right) \geq t\right\}\right| \geq\right.$ $\delta\} \in \mathscr{I}$. This completes the proof.
Definition 24. A sequence $\left\{x_{n}\right\}_{n \in \mathbb{N}}$ in a PM space $X$ is said to be $\mathscr{I}^{*}$-statistically convergent to $x$ if there exists a set $M=$ $\left\{m_{1}<m_{2}<\cdots\right\} \in \mathscr{F}(\mathscr{I})$ such that for all $t>0$,

$$
\lim _{k \rightarrow \infty} \frac{1}{m_{k}}\left|\left\{r \leq m_{k}: x_{r} \notin \mathcal{N}_{x}(t)\right\}\right|=0 .
$$

In this case, we write $x_{n} \rightarrow x\left(S_{\mathrm{PM}}\left(\mathscr{I}^{*}\right)\right)$.

Theorem 25. If a sequence $\left\{x_{n}\right\}_{n \in \mathbb{N}}$ in a PM space $X$ is $\mathscr{I}^{*}$ statistically convergent, then it is strong $\mathscr{I}$-statistically convergent. Moreover, if $\mathscr{I}$ is an admissible ideal satisfying the property $(A P)$, then strong $\mathscr{I}$-statistical convergence implies $\mathscr{I}^{*}$ statistical convergence for any sequence $\left\{x_{n}\right\}_{n \in \mathbb{N}}$ in $X$.

Proof. Let $\left\{x_{n}\right\}_{n \in \mathbb{N}}$ in $X$ be a sequence such that $x_{n} \rightarrow$ $x\left(S_{\mathrm{PM}}\left(\mathscr{I}^{*}\right)\right)$. Then, there exists a set $M=\left\{m_{1}<m_{2}<\cdots\right\} \in$ $\mathscr{F}(\mathscr{I})$ such that

$$
\lim _{k \rightarrow \infty} \frac{1}{m_{k}}\left|\left\{r \leq m_{k}: x_{r} \notin \mathcal{N}_{x}(t)\right\}\right|=0,
$$

that is, for $\delta>0$ there exists a natural number $k_{0}$ such that $\left(1 / m_{k}\right)\left|\left\{r \leq m_{k}: x_{r} \notin \mathcal{N}_{x}(t)\right\}\right|<\delta$ for all $k>k_{0}$. Now, we have

$$
\begin{aligned}
& \left\{n \in \mathbb{N}: \frac{1}{n}\left|\left\{k \leq n: x_{k} \notin \mathcal{N}_{x}(t)\right\}\right| \geq \delta\right\} \\
& \subseteq H \cup\left\{m_{1}, m_{2}, \ldots, m_{k_{0}}\right\},
\end{aligned}
$$

where $H=\mathbb{N} \backslash M \in \mathscr{I}$. Since $\mathscr{I}$ is admissible, the set on the right hand side belongs to $\mathscr{I}$ and consequently $\{n \in \mathbb{N}:(1 /$ $\left.n)\left|\left\{k \leq n: x_{k} \notin \mathscr{N}_{x}(t)\right\}\right| \geq \delta\right\} \in \mathscr{I}$. Hence, $x_{n} \rightarrow$ $x\left(S_{\mathrm{PM}}(\mathscr{I})\right)$.

Next, suppose that $x_{n} \rightarrow x\left(S_{\mathrm{PM}}(\mathscr{I})\right)$. Clearly, the sequence $(1 / n)\left|\left\{k \leq n: x_{k} \notin \mathscr{N}_{x}(t)\right\}\right|$ is $\mathscr{I}$-convergent to zero. Since the ideal $\mathscr{I}$ has the property (AP), the sequence $(1 / n)\left|\left\{k \leq n: x_{k} \notin \mathcal{N}_{x}(t)\right\}\right|$ is $\mathscr{I}^{*}$-convergent to zero. Therefore, $x_{n} \rightarrow x\left(S_{\mathrm{PM}}\left(\mathscr{J}^{*}\right)\right)$.

Definition 26. A sequence $\left\{x_{n}\right\}_{n \in \mathbb{N}}$ in a PM space $X$ is said to be strong $\mathscr{I}$-statistically Cauchy if for every $\epsilon>0$, there exists a positive integer $N=N(\epsilon) \in \mathbb{N}$ such that for any $\delta>0$,

$$
\left\{n \in \mathbb{N}: \frac{1}{n}\left|\left\{k \leq n: x_{k} \notin \mathscr{N}_{x_{N}}(\epsilon)\right\}\right| \geq \delta\right\} \in \mathscr{I} .
$$

Theorem 27. In a PM space $X$ every strong $\mathscr{I}$-statistically convergent sequence is strong $\mathscr{I}$-statistically Cauchy.

Proof. Since $x_{n} \rightarrow x\left(S_{\mathrm{PM}}(\mathscr{I})\right)$, then for any $\epsilon>0$ and $0<$ $\delta<1$,

$$
\left\{n \in \mathbb{N}: \frac{1}{n}\left|\left\{k \leq n: x_{k} \notin \mathscr{N}_{x}\left(\frac{\epsilon}{2}\right)\right\}\right|<\delta\right\} \in \mathscr{F}(\mathscr{I}) .
$$

It follows that

$$
\frac{1}{n}\left|\left\{k \leq n: x_{k} \notin \mathcal{N}_{x}\left(\frac{\epsilon}{2}\right)\right\}\right|<\delta<1 .
$$


Then, there exists a $N \leq n$ such that $x_{N} \in \mathcal{N}_{x}(\epsilon / 2)$. Therefore, $d_{L}\left(F_{x_{N} x}, \varepsilon_{0}\right)<\epsilon / 2$, and so

$$
\begin{aligned}
\{k \leq & \left.n: d_{L}\left(F_{x_{k} x_{N}}, \varepsilon_{0}\right) \geq \epsilon\right\} \\
& \subseteq\left\{k \leq n: d_{L}\left(F_{x_{k} x_{N}}, F_{x_{N} x}\right) \geq \frac{\epsilon}{2}\right\} .
\end{aligned}
$$

Consequently,

$$
\begin{aligned}
& \left\{n \in \mathbb{N}: \frac{1}{n}\left|\left\{k \leq n: d_{L}\left(F_{x_{k} x_{N}}, \varepsilon_{0}\right) \geq \epsilon\right\}\right| \geq \delta\right\} \\
& \quad \subseteq\left\{n \in \mathbb{N}: \frac{1}{n}\left|\left\{k \leq n: d_{L}\left(F_{x_{k} x_{N}}, F_{x_{N} x}\right) \geq \frac{\epsilon}{2}\right\}\right| \geq \delta\right\} \in \mathscr{I} .
\end{aligned}
$$

This completes the proof.

Definition 28. A sequence $\left\{x_{n}\right\}_{n \in \mathbb{N}}$ in $X$ is said to be $\mathscr{I}^{*}$-statistically Cauchy if for every $\epsilon>0$ there exists $N \in \mathbb{N}$ and a set $M=\left\{m_{1}<m_{2}<\cdots\right\} \in \mathscr{F}(\mathscr{I})$ such that

$$
\lim _{k \rightarrow \infty} \frac{1}{m_{k}}\left|\left\{r \leq m_{k}: x_{r} \notin \mathcal{N}_{x_{N}}(\epsilon)\right\}\right|=0 .
$$

Theorem 29. In a PM space $X$, every $\mathscr{I}^{*}$-statistically Cauchy sequence is strong $\mathscr{I}$-statistically Cauchy. Moreover, if $\mathscr{I}$ is an admissible ideal satisfying the property (AP), then strong $\mathscr{I}$ statistically Cauchy sequence coincides with $\mathscr{I}^{*}$-statistically Cauchy sequence.

Proof. The proof is straightforward, and so it has been omitted.

\section{Strong $\mathscr{I}$-Lacunary Statistical Convergence in PM Space}

In this section, we discuss some of the results associated with the lacunary statistical convergence and extend certain summability methods using this notion.

By a lacunary sequence, we mean an increasing integer sequence $\theta=\left\{k_{r}\right\}$ such that $k_{0}=0$ and $h_{r}=k_{r}-k_{r-1} \rightarrow \infty$ as $r \rightarrow \infty$. Throughout this paper, the interval determined by $\theta$ shall be denoted by $I_{r}=\left(k_{r-1}, k_{r}\right]$, and the ratio $k_{r} / k_{r-1}$ shall be denoted by $q_{r}$ [5]. The lacunary sequence $\beta=\left\{l_{r}\right\}$ is said to be the lacunary refinement of the lacunary sequence $\theta=\left\{k_{r}\right\}$ if $\left\{k_{r}\right\} \subseteq\left\{l_{r}\right\}[30]$.

Definition 30. Let $\theta=\left\{k_{r}\right\}$ be a lacunary sequence. A sequence $\left\{x_{n}\right\}_{n \in \mathbb{N}}$ in a PM space $X$ is said to be strong $\mathscr{I}$ lacunary statistically convergent to $x$ if for every $\epsilon>0$ and $\delta>0$,

$$
\left\{r \in \mathbb{N}: \frac{1}{h_{r}}\left|\left\{k \in I_{r}: x_{k} \notin \mathcal{N}_{x}(\epsilon)\right\}\right| \geq \delta\right\} \in \mathcal{I} .
$$

In this case, we write $x_{n} \rightarrow x\left(S_{\mathrm{PM}}^{\theta}(\mathscr{I})\right)$. The class of all strong $\mathscr{I}$-lacunary statistically convergent sequences is simply denoted by $\left(S_{\mathrm{PM}}^{\theta}(\mathscr{I})\right)$.
Theorem 31. In a PM space X, the strong $\mathscr{I}$-lacunary statistical limit of a sequence is unique.

Proof. It is similar to the proof of Theorem 22, and therefore it has been omitted.

Theorem 32. For a sequence $\left\{x_{n}\right\}_{n \in \mathbb{N}}$ in a PM space $X$, the following conditions are equivalent:

(1) $\left\{x_{n}\right\}_{n \in \mathbb{N}}$ is strong $\mathscr{I}$-lacunary statistically convergent to $x$;

(2) for all $\epsilon>0$,

$$
\left\{r \in \mathbb{N}: \frac{1}{h_{r}} \sum_{k \in I_{r}} d_{L}\left(F_{x_{k} x}, \varepsilon_{0}\right) \geq \epsilon\right\} \in \mathscr{I} .
$$

Proof. (1) $\Rightarrow$ (2): First suppose that $x_{n} \rightarrow x\left(S_{\mathrm{PM}}^{\theta}(\mathscr{I})\right)$. Since $d_{L}\left(F_{x_{k} x}, \varepsilon_{0}\right) \leq 1$ for all $k \in \mathbb{N}$ then for $\epsilon>0$, we have

$$
\begin{aligned}
\frac{1}{h_{r}} \sum_{k \in I_{r}} d_{L}\left(F_{x_{k} x}, \varepsilon_{0}\right)= & \frac{1}{h_{r}} \sum_{\substack{k \in I_{r} \\
d_{L}\left(F_{x_{k} l}, \varepsilon_{0}\right) \geq \epsilon / 2}} d_{L}\left(F_{x_{k} x}, \varepsilon_{0}\right) \\
& +\frac{1}{h_{r}} \sum_{k \in I_{r}} d_{L}\left(F_{x_{k} x}, \varepsilon_{0}\right) \\
\leq & \frac{1}{h_{r}\left(F_{x_{k} x}, \varepsilon_{0}\right)<\epsilon / 2}\left|\left\{k \in I_{r}: d_{L}\left(F_{x_{k} x}, \varepsilon_{0}\right) \geq \frac{\epsilon}{2}\right\}\right|+\frac{\epsilon}{2} .
\end{aligned}
$$

Consequently, we have

$$
\begin{aligned}
& \left\{r \in \mathbb{N}: \frac{1}{h_{r}} \sum_{k \in I_{r}} d_{l}\left(F_{x_{k} x}, \varepsilon_{0}\right) \geq \epsilon\right\} \\
& \subseteq\left\{r \in \mathbb{N}: \frac{1}{h_{r}}\left|\left\{k \in I_{r}: d_{L}\left(F_{x_{k} x}, \varepsilon_{0}\right) \geq \varepsilon\right\}\right| \geq \frac{\epsilon}{2}\right\} \in \mathscr{I} .
\end{aligned}
$$

$(2) \Rightarrow(1)$ : Next suppose that condition (2) holds. Now, for $\epsilon>0$, we have

$$
\begin{aligned}
& \sum_{k \in I_{r}} d_{L}\left(F_{x_{k} x}, \varepsilon_{0}\right) \geq \sum_{\substack{k \in I_{r} \\
d_{L}\left(F_{x_{k} x}, \varepsilon_{0}\right) \geq \epsilon}} d_{L}\left(F_{x_{k} x}, \varepsilon_{0}\right) \\
& \geq \epsilon\left|\left\{k \in I_{r}: d_{L}\left(F_{x_{k} x}, \varepsilon_{0}\right) \geq \epsilon\right\}\right| .
\end{aligned}
$$

Therefore,

$$
\frac{1}{\epsilon h_{r}} \sum_{k \in I_{r}} d_{L}\left(F_{x_{k} x}, \varepsilon_{0}\right) \geq \frac{1}{h_{r}}\left|\left\{k \in I_{r}: d_{L}\left(F_{x_{k} x}, \varepsilon_{0}\right) \geq \epsilon\right\}\right| .
$$


Then, for any $\delta>0$, we have

$$
\begin{gathered}
\left\{r \in \mathbb{N}: \frac{1}{h_{r}}\left|\left\{k \in I_{r}: d_{L}\left(F_{x_{k} x}, \varepsilon_{0}\right) \geq \epsilon\right\}\right| \geq \delta\right\} \\
\subseteq\left\{r \in \mathbb{N}: \frac{1}{h_{r}} \sum_{k \in I_{r}} d_{L}\left(F_{x_{k}}, \varepsilon_{0}\right) \geq \varepsilon \delta\right\} \in \mathscr{I} .
\end{gathered}
$$

Therefore, $x_{n} \rightarrow x\left(S_{\mathrm{PM}}^{\theta}(\mathscr{I})\right)$. This completes the proof.

Theorem 33. For any lacunary sequence $\theta=\left\{k_{r}\right\}$, strong $\mathscr{I}$ statistical convergence in a PM space implies strong $\mathscr{I}$-lacunar$y$ statistical convergence if and only if $\lim _{\inf } \rightarrow \infty q_{r}>1$.

Proof. Suppose first that $\liminf _{r \rightarrow \infty} q_{r}>1$. Then, there exists a $\alpha>0$ such that $q_{r} \geq 1+\alpha$ for sufficiently large $r$. This implies that $h_{r} / k_{r} \geq \alpha /(1+\alpha)$. Since $x_{k} \rightarrow x\left(S_{\mathrm{PM}}(\mathscr{I})\right)$, for every $\epsilon>0$ and for sufficiently large $r$, we have

$$
\begin{aligned}
& \frac{1}{k_{r}}\left|\left\{k \leq k_{r}: d_{L}\left(F_{x_{k} x}, \varepsilon_{0}\right) \geq \epsilon\right\}\right| \\
& \quad \geq \frac{1}{k_{r}}\left|\left\{k \in I_{r}: d_{L}\left(F_{x_{k} x}, \varepsilon_{0}\right) \geq \epsilon\right\}\right| \\
& \quad \geq \frac{\alpha}{1+\alpha} \cdot \frac{1}{h_{r}}\left|\left\{k \in I_{r}: d_{L}\left(F_{x_{k} x}, \varepsilon_{0}\right) \geq \epsilon\right\}\right| .
\end{aligned}
$$

Then, for any $\delta>0$, we have

$$
\begin{aligned}
& \left\{r \in \mathbb{N}: \frac{1}{h_{r}}\left|\left\{k \in I_{r}: d_{L}\left(F_{x_{k} x}, \varepsilon_{0}\right) \geq \epsilon\right\}\right| \geq \delta\right\} \\
& \quad \subseteq\left\{r \in \mathbb{N}: \frac{1}{k_{r}}\left|\left\{k \leq k_{r}: d_{L}\left(F_{x_{k} x}, \varepsilon_{0}\right) \geq \epsilon\right\}\right| \geq \frac{\delta \alpha}{1+\alpha}\right\} \in \mathscr{I} .
\end{aligned}
$$

This proves the result.

Let $\mathscr{I}$ be an admissible ideal of $\mathbb{N}$, and let $f: \mathbb{N} \rightarrow \mathbb{N}$ be any function. Define $\mathscr{I}^{f}=\{A \subseteq \mathbb{N}: f(A) \in \mathscr{I}\}$. We can easily show that $\mathscr{I}^{f}$ is also an ideal of $\mathbb{N}$. First, we note that $\emptyset=f(\emptyset) \in \mathscr{I}$ and so $\emptyset \in \mathscr{I}^{f}$. If $A, B \in \mathscr{I}^{f}$, then $f(A \cup B)=$ $f(A) \cup f(B) \in \mathscr{I}$ which implies that $A \cup B \in \mathscr{I}^{f}$. Also if $B \in \mathscr{I}^{f}$ and $A \subseteq B$, then $f(A) \subseteq f(B)$ and $f(B) \in \mathscr{I}$ which in turn implies that $A \in \mathscr{I}^{f}$. Therefore, $\mathscr{I}^{f}$ is an ideal of $\mathbb{N}$.

Theorem 34. Let $\beta=\left\{l_{r}\right\}$ be a lacunary refinement of the lacunary sequence $\theta=\left\{k_{r}\right\}$. Let $I_{r}=\left(k_{r-1}, k_{r}\right]$ and $J_{r}=$ $\left(l_{r-1}, l_{r}\right], r=1,2, \ldots$. If there exists a $\delta>0$ such that

$$
\begin{gathered}
\frac{\left|J_{j}\right|}{\left|I_{i}\right|} \geq \delta \quad \text { for every } J_{j} \subseteq I_{i}, \\
i=f(j) \quad \text { for some function } f: \mathbb{N} \rightarrow \mathbb{N},
\end{gathered}
$$

then $x_{k} \rightarrow x\left(S_{P M}^{\theta}(\mathscr{I})\right)$ implies that $x_{k} \rightarrow x\left(S_{P M}^{\beta}\left(\mathscr{I}^{f}\right)\right)$.
Proof. Let $x_{k} \rightarrow x\left(S_{\mathrm{PM}}^{\theta}(\mathscr{I})\right)$. Therefore,

$$
\left\{r \in \mathbb{N}: \frac{1}{\left|I_{r}\right|}\left|\left\{k \in I_{r}: d_{L}\left(F_{x_{k} x}, \varepsilon_{0}\right) \geq \epsilon\right\}\right| \geq \delta\right\} \in \mathscr{I} .
$$

For $\epsilon>0$ and for every $J_{j}$, we can find $I_{i}$ such that $J_{j} \subseteq I_{i}$. Thus, we obtain

$$
\begin{aligned}
& \frac{1}{\left|J_{j}\right|}\left|\left\{k \in J_{j}: d_{L}\left(F_{x_{k} x}, \varepsilon_{0}\right) \geq \epsilon\right\}\right| \\
& \quad \leq \frac{1}{\delta}\left(\frac{1}{\left|I_{i}\right|}\right)\left|\left\{k \in I_{i}: d_{L}\left(F_{x_{k} x}, \varepsilon_{0}\right) \geq \epsilon\right\}\right| .
\end{aligned}
$$

For $\eta>0$, we have

$$
\begin{aligned}
& \frac{1}{\left|I_{i}\right|}\left|\left\{k \in I_{i}: d_{L}\left(F_{x_{k} x}, \varepsilon_{0}\right) \geq \epsilon\right\}\right| \\
& \quad \geq \frac{\delta}{\left|J_{j}\right|}\left|\left\{k \in J_{j}: d_{L}\left(F_{x_{k} x}, \varepsilon_{0}\right) \geq \epsilon\right\}\right| \geq \eta \delta .
\end{aligned}
$$

Since $x_{k} \rightarrow x\left(S_{\mathrm{PM}}^{\theta}(\mathscr{I})\right)$, there exists some $j \in \mathbb{N}$ such that

A

$$
=\left\{f(j) \in \mathbb{N}: \frac{1}{\left|J_{j}\right|}\left|\left\{k \in J_{j}: d_{L}\left(F_{x_{k} x}, \varepsilon_{0}\right) \geq \epsilon\right\}\right| \geq \eta\right\} \in \mathscr{I} .
$$

Therefore,

$$
\left\{j \in \mathbb{N}: \frac{1}{\left|J_{j}\right|}\left|\left\{k \in J_{j}: d_{L}\left(F_{x_{k} x}, \varepsilon_{0}\right) \geq \epsilon\right\}\right| \geq \eta\right\} \in \mathscr{I}^{f} .
$$

This completes the proof.

\section{Strong $\mathscr{I}$ - $\lambda$-Statistical Convergence in PM Space}

In this section, we introduce the concepts of strong $\mathscr{I}$ - $\lambda$ statistical convergence and $[V, \lambda](\mathscr{I})$-summability in a PM space.

Let $\lambda=\left\{\lambda_{n}\right\}_{n \in \mathbb{N}}$ be a nondecreasing sequence of positive numbers tending to $\infty$ such that $\lambda_{n+1} \leq \lambda_{n}+1, \lambda_{1}=$ 1. The collection of all such sequences $\lambda$ is denoted by $\mathscr{D}$. The generalised de la Vallée-Poussin mean is defined for the sequence $\left\{x_{n}\right\}_{n \in \mathbb{N}}$ of reals by

$$
t_{n}(x)=\frac{1}{\lambda_{n}} \sum_{k \in Q_{n}} x_{k}
$$

where $Q_{n}=\left[n-\lambda_{n}+1, n\right]$. A sequence $\left\{x_{n}\right\}_{n \in \mathbb{N}}$ of reals is said to be $[V, \lambda](\mathscr{I})$ summable to $x$ if $\mathscr{I}$ - $\lim t_{n}(x)=x$, that is, for $\epsilon>0,\left\{n \in \mathbb{N}:\left|t_{n}(x)-x\right| \geq \epsilon\right\} \in \mathscr{I}[13,31]$.

We now introduce the main definitions for this section. 
Definition 35. Any sequence $\left\{x_{n}\right\}_{n \in \mathbb{N}}$ in a PM space $X$ is said to be strong $\mathscr{I}$ - $\lambda$-statistically convergent to $x$ if for every $\epsilon>$ 0 and $\delta>0$,

$$
\left\{n \in \mathbb{N}: \frac{1}{\lambda_{n}}\left|\left\{k \in Q_{n}: d_{L}\left(F_{x_{k} x}, \varepsilon_{0}\right) \geq \epsilon\right\}\right| \geq \delta\right\} \in \mathscr{I} .
$$

In this case, we write $x_{n} \rightarrow x\left(S_{\mathrm{PM}}^{\lambda}(\mathscr{I})\right)$. The collection of all such sequences is simply denoted by $\left(S_{\mathrm{PM}}^{\lambda}(\mathscr{I})\right)$.

Definition 36. Any sequence $\left\{x_{n}\right\}_{n \in \mathbb{N}}$ in a PM space $X$ is said to be $[V, \lambda](\mathscr{I})$-summable to $x$ in $X$ if for every $\delta>0$,

$$
\left\{n \in \mathbb{N}: \frac{1}{\lambda_{n}} \sum_{k \in Q_{n}} d_{L}\left(F_{x_{k} x}, \varepsilon_{0}\right) \geq \delta\right\} \in \mathscr{I} .
$$

In this case, we write $x_{n} \rightarrow x[V, \lambda]_{\mathrm{PM}}(\mathscr{I})$. The collection of all such sequences is simply denoted by $[V, \lambda]_{\mathrm{PM}}(\mathscr{I})$.

Theorem 37. For any sequence $\left\{x_{n}\right\}_{n \in \mathbb{N}}$ in a PM space $X$, the following conditions are equivalent.

(1) The sequence $\left\{x_{n}\right\}_{n \in \mathbb{N}}$ is strong $\mathscr{I}$ - $\lambda$-statistically convergent to $x$ in $X$.

(2) The sequence $\left\{x_{n}\right\}_{n \in \mathbb{N}}$ is $[V, \lambda](\mathscr{I})$ summable to $x$ in $X$.

Proof. It is similar to the proof of Theorem 32, and therefore it has been omitted.

Example 38. Consider that $X=\mathbb{R}$ with the usual metric $d$ and $G(x)=1-e^{-x}$. Clearly, $G \in \Delta^{+}$. We define $\mathfrak{F}: X \times X \rightarrow$ $\Delta^{+}$by $\mathfrak{F}(x, y)(t)=F_{x y}(t)=G(t / d(x, y))=1-e^{-t /|x-y|}$ for all $x, y \in X$. Let us define $G(x / 0)=G(\infty)=1$ for $x>0$ and $G(0 / 0)=G(0)=0$. Clearly, $(X, \mathfrak{F}, \tau)$ is a PM space where $\tau$ is the continuous triangle function. Recall that for any $t>0$, $F(t)>1-t$ if and only if $d_{L}\left(F, \varepsilon_{0}\right)<t[24]$.

Let $\mathscr{I}$ be an admissible ideal of $\mathbb{N}$ and $\lambda_{n} \in \mathscr{D}$. Take a fixed $A \in \mathscr{I}$ and define a sequence $\left\{x_{k}\right\}_{k \in \mathbb{N}}$ in $X$ by

$$
x_{k}= \begin{cases}k u, & \text { for } n-\left[\sqrt{\lambda_{n}}\right]+1 \leq k \leq n, n \notin A \\ k u, & \text { for } n-\lambda_{n}+1 \leq k \leq n, n \in A,\end{cases}
$$

where $u$ is a fixed element in $X$ with $|u|=1$. Clearly, $F_{x_{k} 0}(t)=$ $1-e^{-t /\left|x_{\mathrm{k}}\right|}=1-e^{-t / k}$. Now, for every $\epsilon>0(0<\epsilon<1)$, there exists a $k_{0} \in \mathbb{N}$ such that $F_{x_{k} 0}(\varepsilon) \leq 1-\epsilon$ for all $k \geq k_{0}$. Therefore, for every $\delta>0$,

$$
\frac{1}{\lambda_{n}}\left|\left\{k \in Q_{n}: d_{L}\left(F_{x_{k} 0}, \varepsilon_{0}\right) \geq \epsilon\right\}\right|=\frac{\left[\sqrt{\lambda_{n}}\right]}{\lambda_{n}} \longrightarrow 0,
$$

as $n \rightarrow \infty$ and $n \notin A$. Consequently, for every $\delta>0$,

$$
\begin{aligned}
& \left\{n \in \mathbb{N}: \frac{1}{\lambda_{n}}\left|\left\{k \in Q_{n}: d_{L}\left(F_{x_{k} 0}, \varepsilon_{0}\right) \geq \epsilon\right\}\right| \geq \delta\right\} \\
& \subseteq A \cup\{1,2, \ldots, m\},
\end{aligned}
$$

for some $m \in \mathbb{N}$. Since $\mathscr{I}$ is admissible, the expression on the right hand side belongs to $\mathscr{I}$. It follows that $x_{k} \rightarrow$ $0\left(S_{\mathrm{PM}}^{\lambda}(\mathscr{I})\right)$.
Theorem 39. If $\liminf _{n \rightarrow \infty}\left(\lambda_{n} / n\right)>0$, then $S_{P M}(\mathscr{I}) \subseteq$ $S_{P M}^{\lambda}(\mathscr{I})$.

Proof. For $\epsilon>0$, we have

$$
\begin{aligned}
& \frac{1}{n}\left|\left\{k \leq n: d_{L}\left(F_{x_{k} x}, \varepsilon_{0}\right) \geq \epsilon\right\}\right| \\
& \geq \frac{1}{n}\left|\left\{k \in Q_{n}: d_{L}\left(F_{x_{k} x}, \varepsilon_{0}\right) \geq \epsilon\right\}\right| \\
& \quad=\frac{\lambda_{n}}{n} \frac{1}{\lambda_{n}}\left|\left\{k \in Q_{n}: d_{L}\left(F_{x_{k} x}, \varepsilon_{0}\right) \geq \epsilon\right\}\right| .
\end{aligned}
$$

If $\liminf _{n \rightarrow \infty}\left(\lambda_{n} / n\right)=a>0$, then from definition $\{n \in \mathbb{N}$ : $\left.\lambda_{n} / n<a / 2\right\}$ is finite. Thus, for $\delta>0$,

$$
\begin{aligned}
& \left\{n \in \mathbb{N}: \frac{1}{\lambda_{n}}\left|\left\{k \in Q_{n}: d_{L}\left(F_{x_{k} x}, \varepsilon_{0}\right) \geq \epsilon\right\}\right| \geq \delta\right\} \\
& \subseteq\left\{n \in \mathbb{N}: \frac{1}{n}\left|\left\{k \in Q_{n}: d_{L}\left(F_{x_{k} x}, \varepsilon_{0}\right) \geq \epsilon\right\}\right| \geq \frac{a}{2} \delta\right\} \\
& \cup\left\{n \in \mathbb{N}: \frac{\lambda_{n}}{n}<\frac{a}{2}\right\} .
\end{aligned}
$$

Since $\mathscr{I}$ is admissible, the set on the right hand side belongs to $\mathscr{I}$, and this completes the proof.

Theorem 40. Let $\lambda \in \mathscr{D}$ be such that $\lim _{n \rightarrow \infty}\left(\lambda_{n} / n\right)=1$. Then, $S_{P M}^{\lambda}(\mathscr{I}) \subset S_{P M}(\mathscr{I})$.

Proof. Let $\delta>0$ be given. Since $\lim _{n \rightarrow \infty}\left(\lambda_{n} / n\right)=1$, we can choose $m \in \mathbb{N}$ such that $\left|\lambda_{n} / n-1\right|<\delta / 2$ for all $n \geq m$. Now, observe that for $\epsilon>0$,

$$
\begin{aligned}
\frac{1}{n}\left|\left\{k \leq n: d_{L}\left(F_{x_{k} x}, \varepsilon_{0}\right) \geq \epsilon\right\}\right| \\
=\frac{1}{n}\left|\left\{k \leq n-\lambda_{n}: d_{L}\left(F_{x_{k} x}, \varepsilon_{0}\right) \geq \epsilon\right\}\right| \\
\quad+\frac{1}{n}\left|\left\{k \in Q_{n}: d_{L}\left(F_{x_{k} x}, \varepsilon_{0}\right) \geq \epsilon\right\}\right| \\
\leq \frac{n-\lambda_{n}}{n}+\frac{1}{n}\left|\left\{k \leq Q_{n}: d_{L}\left(F_{x_{k} x}, \varepsilon_{0}\right) \geq \epsilon\right\}\right| \\
\leq 1-\left(1-\frac{\delta}{2}\right)+\frac{1}{n}\left|\left\{k \in Q_{n}: d_{L}\left(F_{x_{k} x}, \varepsilon_{0}\right) \geq \epsilon\right\}\right| \\
=\frac{\delta}{2}+\frac{1}{n}\left|\left\{k \leq Q_{n}: d_{L}\left(F_{x_{k} x}, \varepsilon_{0}\right) \geq \epsilon\right\}\right|,
\end{aligned}
$$

for all $n \geq m$. Hence,

$$
\begin{aligned}
& \left\{n \in \mathbb{N}: \frac{1}{n}\left|\left\{k \leq n: d_{L}\left(F_{x_{k} x}, \varepsilon_{0}\right) \geq \epsilon\right\}\right| \geq \delta\right\} \\
& \subseteq\left\{n \in \mathbb{N}: \frac{1}{n}\left|\left\{k \in Q_{n}: d_{L}\left(F_{x_{k} x}, \varepsilon_{0}\right) \geq \epsilon\right\}\right| \geq \frac{\delta}{2}\right\} \\
& \cup\{1,2, \ldots, m\} .
\end{aligned}
$$

If $x_{k} \rightarrow x\left(S_{\mathrm{PM}}^{\lambda}(\mathscr{I})\right)$, then the set on the right hand side belongs to $\mathscr{I}$ and so the left hand side also belongs to $\mathscr{I}$. This shows that $x_{k} \rightarrow x\left(S_{\mathrm{PM}}(\mathscr{I})\right)$. 
Remark 41. Consider the sequence $\left\{\lambda_{n}\right\}_{n \in \mathbb{N}}$ where $\lambda_{n}=1$ for $n=1,2, \ldots, 10$ and $\lambda_{n}=n-10$ for all $n \geq 10$. Construct the sequence as in Example 38. For $\mathscr{I}=\mathscr{I}_{d}$ (the ideal of density zero sets in $\mathbb{N}$ ) and $A=\left\{1^{2}, 2^{2}, 3^{2}, \ldots\right\}$, the sequence $\left\{x_{k}\right\}_{k \in \mathbb{N}}$ is an example of a sequence which is strong $\mathscr{I}$-statistically convergent but is not statistically convergent in a PM space.

\section{References}

[1] H. Fast, "Sur la convergence statistique," vol. 2, pp. 241-244, 1951.

[2] J. S. Connor, "The statistical and strong $p$-Cesàro convergence of sequences," Analysis, vol. 8, no. 1-2, pp. 47-63, 1988.

[3] J. A. Fridy, "On statistical convergence," Analysis, vol. 5, no. 4, pp. 301-313, 1985.

[4] T. Šalát, "On statistically convergent sequences of real numbers," Mathematica Slovaca, vol. 30, no. 2, pp. 139-150, 1980.

[5] J. A. Fridy and C. Orhan, "Lacunary statistical convergence," Pacific Journal of Mathematics, vol. 160, no. 1, pp. 43-51, 1993.

[6] J. Li, "Lacunary statistical convergence and inclusion properties between lacunary methods," International Journal of Mathematics and Mathematical Sciences, vol. 23, no. 3, pp. 175-180, 2000.

[7] Mursaleen, " $\lambda$-statistical convergence," Mathematica Slovaca, vol. 50, no. 1, pp. 111-115, 2000.

[8] V. Karakaya, N. Şimşek, M. Ertürk, and F. Gürsoy, "Lacunary statistical convergence of sequences of functions in intuitionistic fuzzy normed space," Journal of Intelligent \& Fuzzy Systems. In press.

[9] V. Karakaya, N. Şimşek, M. Ertürk, and F. Gürsoy, " $\lambda$-statistical convergence of sequences of functions in intuitionistic fuzzy normed spaces," Journal of Function Spaces and Applications, vol. 2012, Article ID 926193, 14 pages, 2012.

[10] V. Karakaya, N. Şimşek, M. Ertürk, and F. Gürsoy, "Lacunary statistical convergence of sequences of functions in intuitionistic fuzzy normed space," Journal of Intelligent \& Fuzzy Systems. In press.

[11] P. Kostyrko, T. Šalát, and W. Wilczyński, “ $\mathscr{I}$-convergence," Real Analysis Exchange, vol. 26, no. 2, pp. 669-685, 2000.

[12] P. Das, E. Savas, and S. Kr. Ghosal, "On generalizations of certain summability methods using ideals," Applied Mathematics Letters, vol. 24, no. 9, pp. 1509-1514, 2011.

[13] E. Savas and P. Das, "A generalized statistical convergence via ideals," Applied Mathematics Letters, vol. 24, no. 6, pp. 826-830, 2011.

[14] K. Dems, "On $\mathscr{I}$-Cauchy sequences," Real Analysis Exchange, vol. 30, no. 1, pp. 123-128, 2004/05.

[15] A. Nabiev, S. Pehlivan, and M. Gürdal, "On $\mathscr{I}$-Cauchy sequences," Taiwanese Journal of Mathematics, vol. 11, no. 2, pp. 569576, 2007.

[16] P. Das and S. Kr. Ghosal, "Some further results on $\mathscr{I}$-Cauchy sequences and condition (AP)," Computers \& Mathematics with Applications, vol. 59, no. 8, pp. 2597-2600, 2010.

[17] M. Mursaleen and S. A. Mohiuddine, "On ideal convergence in probabilistic normed spaces," Mathematica Slovaca, vol. 62, no. 1, pp. 49-62, 2012.

[18] K. Menger, "Statistical metrics," Proceedings of the National Academy of Sciences of the United States of America, vol. 28, pp. 535-537, 1942.

[19] B. Schweizer and A. Sklar, "Statistical metric spaces," Pacific Journal of Mathematics, vol. 10, pp. 313-334, 1960.
[20] B. Schweizer, A. Sklar, and E. Thorp, "The metrization of statistical metric spaces," Pacific Journal of Mathematics, vol. 10, pp. 673-675, 1960.

[21] B. Schweizer and A. Sklar, "Statistical metric spaces arising from sets of random variables in Euclidean $n$-space," Akademija Nauk SSSR, vol. 7, pp. 456-465, 1962.

[22] B. Schweizer and A. Sklar, "Triangle inequalities in a class of statistical metric spaces," Journal of the London Mathematical Society, vol. 38, pp. 401-406, 1963.

[23] R. M. Tardiff, “Topologies for probabilistic metric spaces," Pacific Journal of Mathematics, vol. 65, no. 1, pp. 233-251, 1976.

[24] B. Schweizer and A. Sklar, Probabilistic Metric Spaces, Dover, Mineola, NY, USA, 2005.

[25] C. Şençimen and S. Pehlivan, "Strong statistical convergence in probabilistic metric spaces," Stochastic Analysis and Applications, vol. 26, no. 3, pp. 651-664, 2008.

[26] C. Şençimen and S. Pehlivan, "Strong ideal convergence in probabilistic metric spaces," Indian Academy of Sciences, vol. 119, no. 3, pp. 401-410, 2009.

[27] C. Şençimen and S. Pehlivan, "Statistical continuity in probabilistic normed spaces," Applicable Analysis, vol. 87, no. 3, pp. 377-384, 2008.

[28] C. Şençimen and S. Pehlivan, "Statistically D-bounded sequences in probabilistic normed spaces," Applicable Analysis, vol. 88, no. 8, pp. 1133-1142, 2009.

[29] D. A. Sibley, "A metric for weak convergence of distribution functions," The Rocky Mountain Journal of Mathematics, vol. 1, no. 3, pp. 427-430, 1971.

[30] A. R. Freedman, J. J. Sember, and M. Raphael, "Some Cesàrotype summability spaces," Proceedings of the London Mathematical Society. Third Series, vol. 37, no. 3, pp. 508-520, 1978.

[31] L. Leindler, "Über die verallgemeinerte de la Vallée-Poussinsche Summierbarkeit allgemeiner Orthogonalreihen," Acta Mathematica Academiae Scientiarum Hungaricae, vol. 16, pp. 375-387, 1965. 


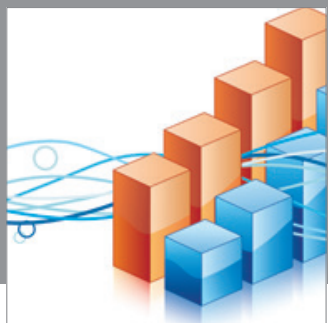

Advances in

Operations Research

mansans

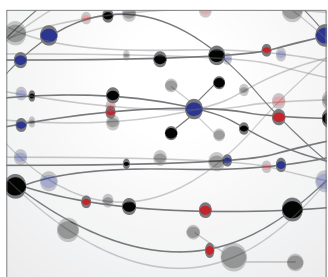

The Scientific World Journal
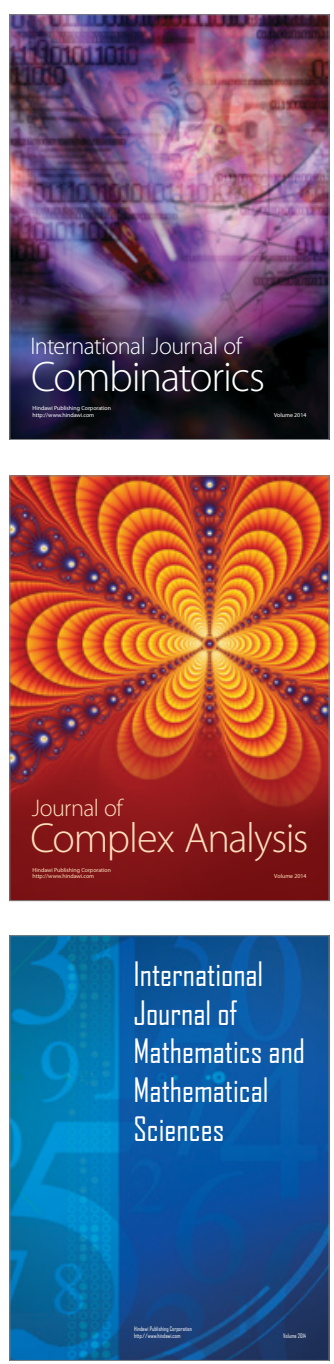
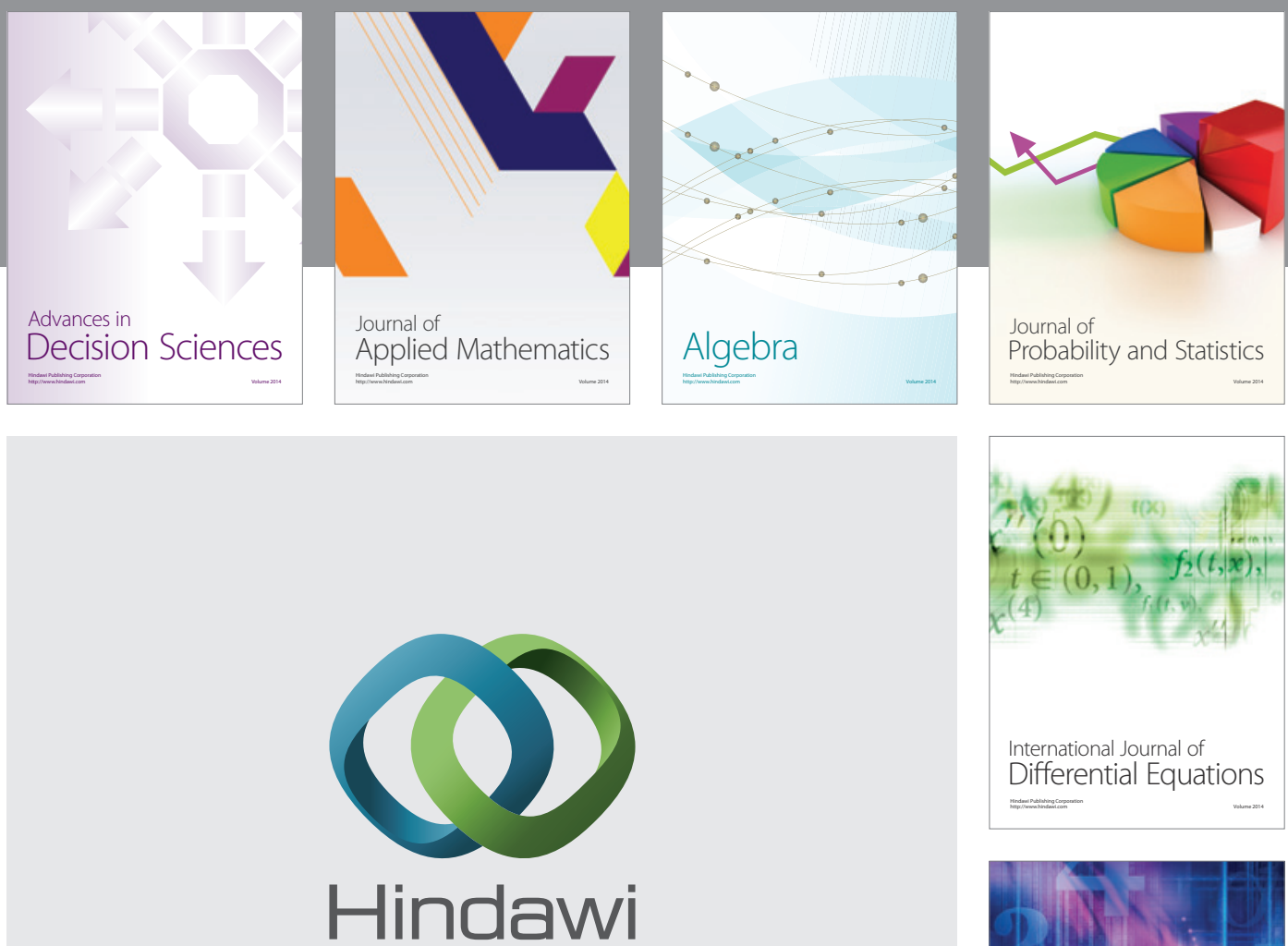

Submit your manuscripts at http://www.hindawi.com
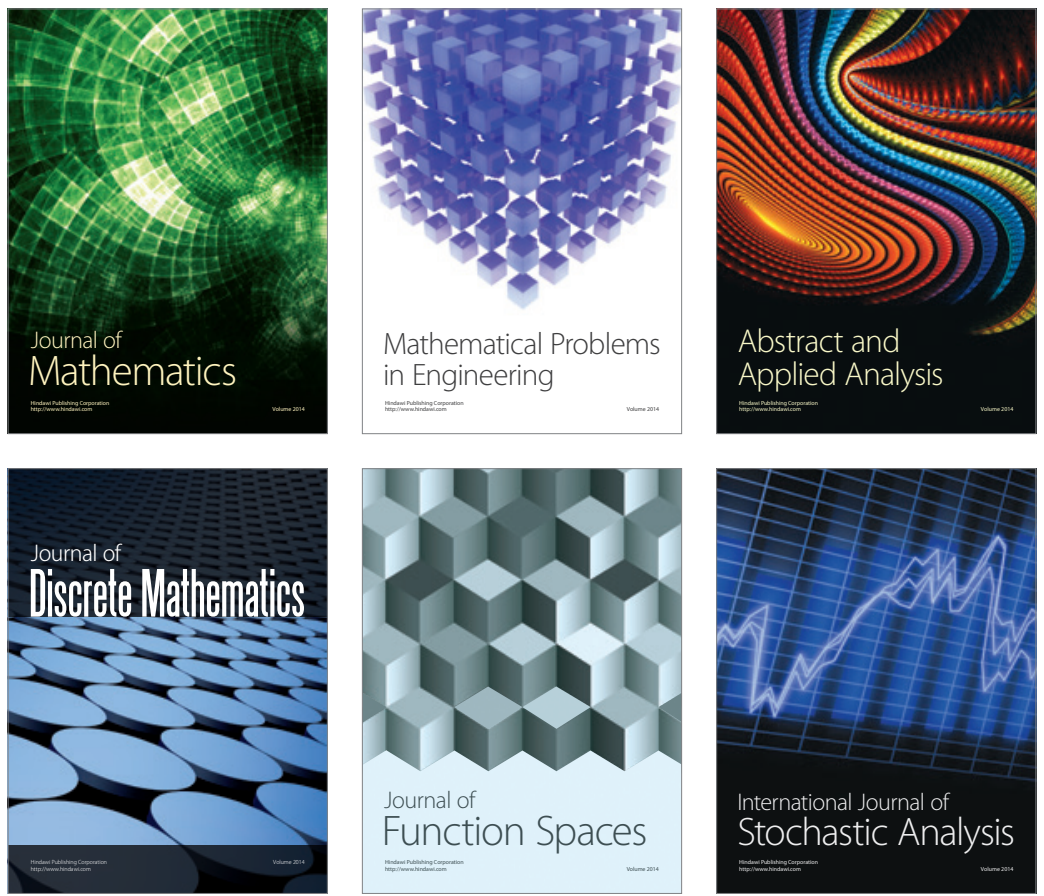

Journal of

Function Spaces

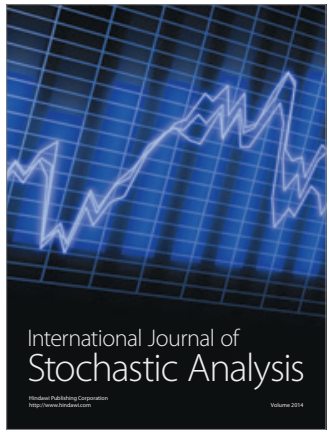

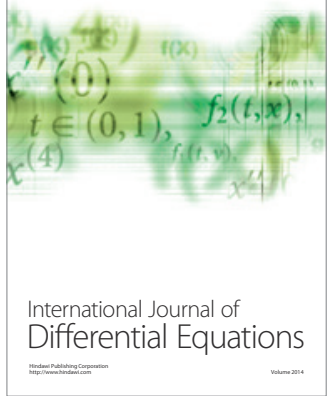
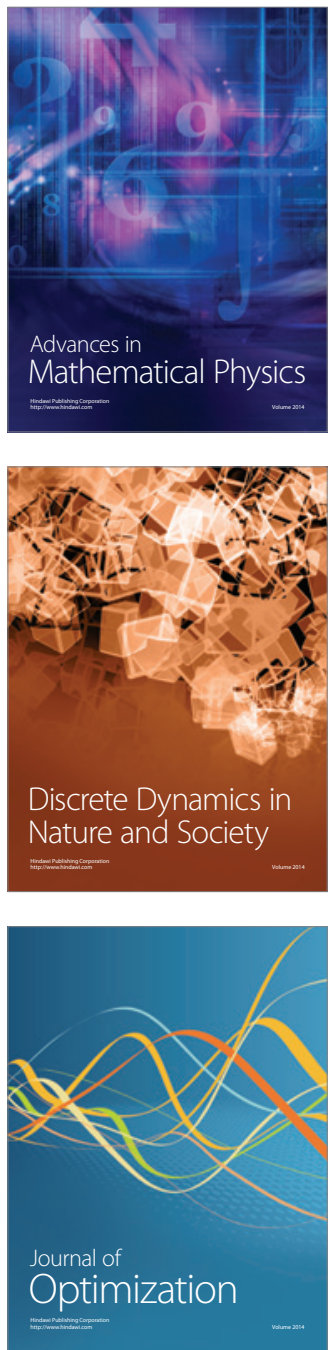